\title{
ELDERLY-SPECIFIC REVISIONS OF DRUG PRESCRIBING INFORMATION IN THE POSTMARKETING ENVIRONMENT IN JAPAN
}

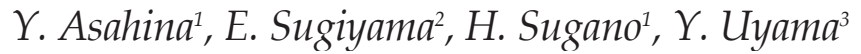

\begin{abstract}
Purpose: This study aimed to characterize elderly-specific revisions of prescribing information of a drug based on recent cases observed in postmarketing use in Japan. Methods: We analyzed all revisions of package inserts that were officially registered between the fiscal year (FY) 1998 and FY 2012. They were examined in terms of a description of the drug, therapeutic area for which the drug is targeted, the period between launch and revision, contents of the revisions, and the evidence sources of safety issues. Results: Of a total of 3,440 revisions occurring between FY 1998 and FY 2012, 2.1\% were specific to drug use in the elderly. Drugs treating the "cardiovascular system" and a miscellaneous category of "various," showed a higher frequency of revisions for elderly patients than those for non-elderly patients. Of elderly-specific revisions occurring between FY 2004 and 2012, the majority were triggered by spontaneous reports or regulatory action in other countries, although there were relatively a few examples of serious safety concerns being raised after initial drug approval. Conclusions: It is important that alternative approaches such as pharmacometrics or pharmacoeoidemiology should be more utilized in Japan for the early and better detection and evaluation of drug safety in elderly patients, especially for therapeutic areas that demonstrate increased susceptibility for risk among the elderly, such as drugs related to the cardiovascular system.
\end{abstract}

Key words: Older patient, Japan, postmarketing safety.

\section{Introduction}

There has been a remarkable increase in the proportion of the elderly population in Japan over the last few decades as well as in other developed countries, and this trend is expected to continue worldwide into the foreseeable future (1). Compared to the wider population, previous studies have shown that the elderly require a considerable amount of prescription drugs $(2,3)$ but the effects of aging, such as increased polypharmacy, comorbidity, cognitive impairment, social problems, and physiological changes affecting pharmacokinetics and pharmacodynamics of drugs $(4,5)$, often complicate the proper evaluation of the efficacy and safety of the drugs used. As a result, the elderly are at an increased risk of developing an adverse drug reaction (ADR). Despite this risk, premarketing clinical trials of many medicines often excluded the elderly to some degree (6-8) with the

1. Office of New Drug II, Pharmaceuticals and Medical Devices Agency (PMDA); 2. Office of Planning and Coordination, Pharmaceuticals and Medical Devices Agency (PMDA); 3. Office of Medical Informatics and Epidemiology, Pharmaceuticals and Medical Devices Agency (PMDA)

Corresponding Author: Yasuko Asahina, Office of New Drug II, Pharmaceuticals and Medical Devices Agency, 3-3-2 Kasumigaseki Chiyodaku Tokyo 100-0013 Japan, Tel: 3-3506-9451, Fax: 3-3506-9453, asahina-yasuko@pmda.go.jp outcome that drugs prescribed to this demographic are regularly approved based on studies involving younger patients.

The lack of data from clinical trials that specifically target the efficacy and safety of new drugs in the elderly may be a barrier to the optimal use of drugs within this population (9-11). ICH E7 guidelines implemented in 1993 (12) and its subsequent Questions and Answers released in 2010 (13), recommend including a meaningful number (at least 100) of patients aged 65 years or older in clinical trials in order to increase the likelihood of detecting clinically important differences between elderly and younger patients. Although the inclusion of elderly patients in such trials has improved since the publication of the ICH E7 guidelines, the number of patients aged 75 years and over typically remains low (14) despite evidence that shows that ADRs are more likely to be severe and responsible for considerable morbidity and mortality for the elderly (15). Given this danger, data pertaining to drug safety in the elderly should be continuously gathered through postmarketing surveillance and risk assessment programs. However, little study examining the difference of safety issues after approval between the elderly and the non-elderly, and issues to be paid more attention regarding drug use in 
the elderly after approval has been reported.

This study aimed to investigate postmarketing drug safety for the elderly by reviewing revisions of package inserts and characterizing elderly-specific issues added after approval in Japan.

\section{Method}

We analyzed all revisions of package inserts that were officially registered between the fiscal year (FY) 1998 and FY 2012 and freely available through the Japanese Pharmaceutical and Medical Devices Agency (PMDA) website. "Specific to the elderly" was defined through inclusion of the term "the elderly" in revisions, or through specific reference to an age bracket that including 65 years and above. Non-prescription drugs and test reagents were excluded from analysis in order to focus on the effects of prescription medicine.

The data collected for all revisions consisted of a description of the drug, the therapeutic area for which the drug is targeted, year of launch, content of revision, year of publication of revision, and the time period between launch and revision publication. For the purpose of our analysis, the therapeutic areas were categorized according to the anatomical therapeutic chemical classification system, with the exception of prescription traditional herbal medicine that was classified as "herb medicine (Kampo)." The time period between launch and revision of package inserts was categorized into (a) less than one year, (b) one-eight years and (c) more than eight years, since the majority of new drugs in Japan undergo a re-examination eight years after approval. The above data was also collected for package insert revisions triggered by safety concerns that were not specific to the elderly in order to enable contrast between the two data sets.

For package insert revisions occurring after the PMDA was established in FY 2004, the source of any corresponding safety concerns were additionally investigated. For all items, the revised information for package inserts was categorized into four groups: (a) new alert for unknown issue, (b) new alert specific to the elderly regarding known issue, (c) elevation of alert level of known issue on the elderly, and (d) additional information for known issue on the elderly. The issues within package inserts were graded into six alert levels according to the degree of importance assigned to the cautions for use in clinical practice as has been previously reported (16). The evidence source of safety issues were categorized into five groups: (a) spontaneous reports in Japan, (b) regulatory action in other countries, (c) review / study/clinical trials by a Marketing Authorization Holder $(\mathrm{MAH}),(\mathrm{d})$ other (i.e., research papers or a revision of package inserts for similar drugs), and (e) more than one source.

\section{Results}

Out of a total of 3,440 revisions of package inserts issued in Japan between FY 1998 and FY 2012, 73 (2.1\%) were specific to drug use in the elderly, ranging from a low of 0.4\% in FY2010 to a high of 9.8\% in FY 2008. The percentages of revisions specific to the elderly to

Table 1

The basic characteristics of the revisions of package inserts between FY 1998 and FY 2012

\begin{tabular}{lllll}
\hline Therapeutic area & \multicolumn{2}{c}{$\begin{array}{c}\text { Specific to the elderly } \\
(\mathbf{n}=\mathbf{7 3})\end{array}$} & $\begin{array}{c}\text { Non-specific to the elderly } \\
(\mathbf{n}=3,367)\end{array}$ \\
\hline Alimentary tract and metabolism & 6 & $8.2 \%$ & 248 & $7.4 \%$ \\
Blood and blood forming organs & 1 & $1.4 \%$ & 156 & $4.6 \%$ \\
Cardiovascular system & 20 & $27.4 \%$ & 329 & $9.8 \%$ \\
Dermatologicals & 0 & $0.0 \%$ & 24 & $0.7 \%$ \\
Genito-urinary system and sex hormones & 0 & $0.0 \%$ & 149 & $4.4 \%$ \\
Systemic hormonal preparations, excluding sex hormones and insulins & 0 & $0.0 \%$ & 66 & $2.0 \%$ \\
Anti-infectives for systemic use & 14 & $19.2 \%$ & 700 & $20.8 \%$ \\
Antineoplastic and immunomodulating agents & 1 & $1.4 \%$ & 583 & $17.3 \%$ \\
Musculo-skeletal system & 0 & $0.0 \%$ & 213 & $6.3 \%$ \\
Nervous system & 17 & $23.3 \%$ & 530 & $15.7 \%$ \\
Antiparasitic products, insecticides and repellents & 0 & $0.0 \%$ & 11 & $0.3 \%$ \\
Respiratory system & 1 & $1.4 \%$ & 142 & $4.2 \%$ \\
Sensory organs & 0 & $0.0 \%$ & 32 & $1.0 \%$ \\
Various & 13 & $17.8 \%$ & 139 & $4.1 \%$ \\
Herb medicine (Kampo) & 0 & $0.0 \%$ & 45 & $1.3 \%$ \\
\hline
\end{tabular}


Table 2

The period between launch and publication of revision of package inserts between FY 1998 and FY 2012

\begin{tabular}{llccc}
\hline Period between launch and publication of revision & & $\begin{array}{c}\text { Specific to the elderly } \\
(\mathbf{n}=\mathbf{7 3})\end{array}$ & $\begin{array}{c}\text { Non-specific to the elderly } \\
(\mathbf{n}=3,367)\end{array}$ \\
\hline$<1$ year & 7 & $9.6 \%$ & 1999 & $5.9 \%$ \\
$1-8$ years & 25 & $34.2 \%$ & 1082 & $32.1 \%$ \\
$>8$ years & 41 & $56.2 \%$ & 2086 & $62 \%$ \\
\hline
\end{tabular}

all revisions were relatively high in FY 1998 (4.1\%), FY 2003 (4.9\%), and FY 2008 (9.8\%) due to an abundance of revisions related to changes in drug class at these times.

As shown in Table 1, "Anti-infectives for systemic use" was common therapeutic area for the revisions with no major difference between those specific and non-specific to the elderly. "Nervous system" was also common but with a little higher frequency for specific to the elderly. Major difference with higher frequency for the revisions specific to the elderly was found in the therapeutic areas of "cardiovascular system" and "various". Drugs labeled under "various" included contact laxatives used to prepare the large bowel before colonoscopy and chelate agents. In contrast, elderly-specific revisions were rare for "the antineoplastic and immunomodulating drugs".

Over half $(56.2 \%)$ of revisions specific to the elderly occurred more than eight years after drug launch, with $34.2 \%$ occurring between one to eight years, and 9.6\% occurring within the first year. A similar tendency was seen among overall revisions, not just those specific to the elderly. This is summarized in Table 2.

\section{Figure 1}

Content of package insert revisions between FY 2004 and FY 2012

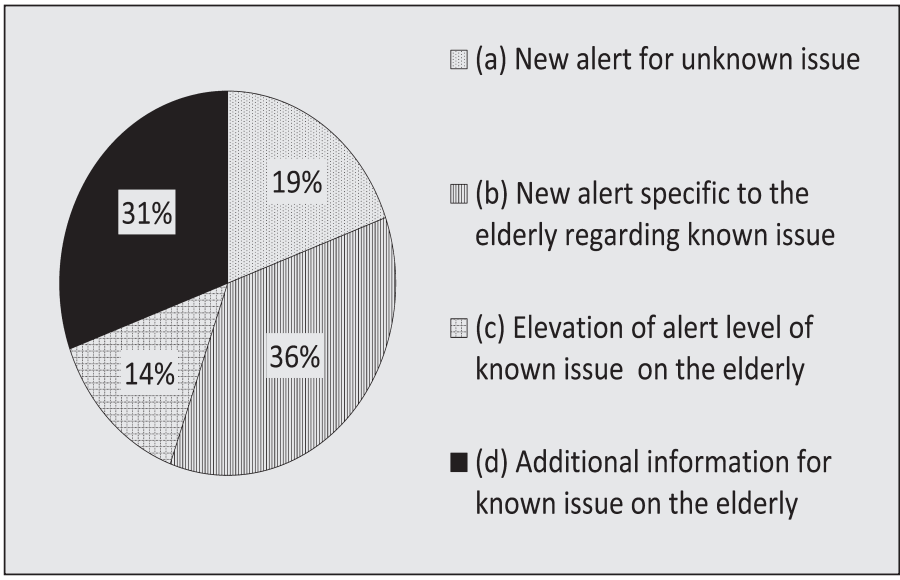

(A) Specific to the elderly $(n=36)$. (B) Non-specific to the elderly $(n=1,527)$

Out of 1,563 notifications of package insert revisions between FY 2004 and FY 2012, 36 (2.3\%) were specific to drug use in the elderly. The proportion of completely novel safety issues within this group was reasonably low $(19 \%, n=7)$, though these seemed to be for typically elderly-specific concerns, such as ileus, aspiration, and dementia like symptoms. Most (5 out of 7) of these revisions were issued more than eight years after initial approval and were based on spontaneous reports or regulatory action in other countries. In addition to highlighting the proportion of elderly-specific revisions that were prompted by new safety concerns, Figure 1 (A) also shows that about half of the elderly-specific revisions $((\mathrm{b})+(\mathrm{c}))$ were related to an increase in existing risk for known issues. The number of cases included in the category (d), "additional information," was almost the same as those in category (b), "new alert specific to the elderly," although the majority (9 out of 11) of cases included within category (d) consisted of revisions that were related to revisions prompted by drug class changes.

\section{Figure 2}

Sources of safety concerns

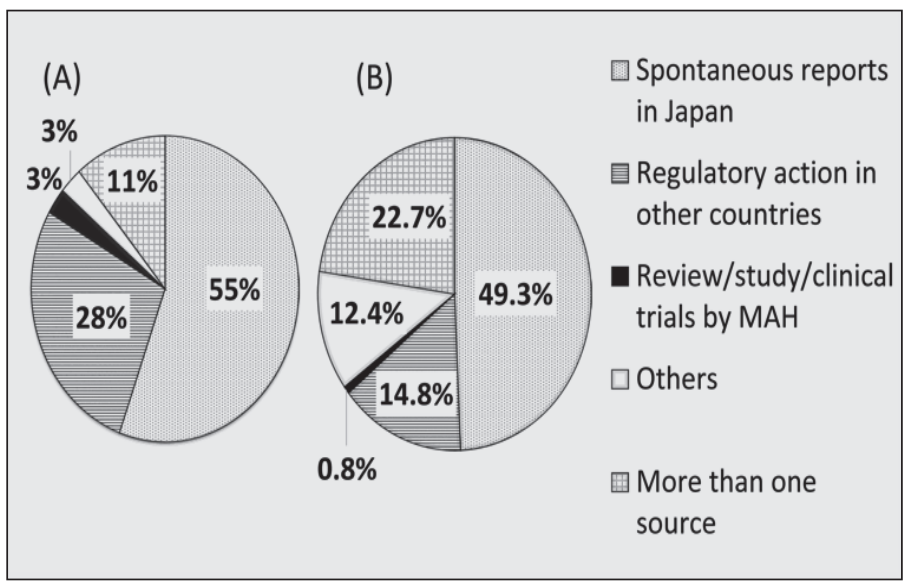

(A) Specific to the elderly $(\mathrm{n}=36)$ (B) Non-specific to the elderly $(\mathrm{n}=1,527)$

For revisions non-specific to the elderly shown in Figure 1 (B), content change for revisions included in the category "additional information for known issue" accounted for $44.1 \%(n=673)$ of all revisions that were non-specific to the elderly identified between FY 2004 and FY 2012 ( $\mathrm{n}=1,527)$, followed by "new alert for unknown issue" with $29.9 \%(\mathrm{n}=456)$ and "elevation of alert level of known issue" at $26.1 \%(\mathrm{n}=398)$.

The evidence sources for both specific to the elderly and non-specific to the elderly safety issues are shown in Figure 2 (A) and (B) respectively. For elderly- 
specific revisions of package inserts, $83 \%$ were based on spontaneous reports or regulatory action in other countries. Academic research accounted for most of the revision included in the category "others" which accounted for $3 \%$ of elderly-specific revisions, but a much larger $12.4 \%$ of non-specific to the elderly revisions. For revisions non-specific to the elderly, the category "more than one source" $(22.7 \%, \mathrm{n}=346)$ consisted mostly of a combination of "spontaneous reports" and "regulatory action in other countries" $(16.0 \%, \mathrm{n}=216)$, but also included "spontaneous reports" and "others" $(3.6 \%, \mathrm{n}=49)$, and "regulatory action in other countries" and "others" $(2.9 \%, \mathrm{n}=39)$. In contrast, the amount of revisions categorized into "more than one source" was very small for elderly-specific cases $(n=4)$.

\section{Discussion}

For revisions specific to the elderly, the therapeutic areas of "cardiovascular system" and "various" underwent relatively frequent package insert revision. Drugs for the areas of "cardiovascular system" detected in this study were used to treat hypertension, hypercholesterolemia and tachyarrhythmia, and adverse reactions developed to these drugs were hyperkalemia, hypokalemia, cardiac arrest and difficulty in swallowing. Given the type of medication included in these categories, this would be expected as these drugs are most commonly used among older patients (2), although several studies have revealed that the inclusion of sufficient numbers of the elderly in clinical trials testing cardiovascular medication is not yet satisfactory (17-19). Given the prevalence of package insert revision in this area, there is a strong case that new drugs are not being evaluated thoroughly enough using samples that include an adequate of elderly patients before obtaining market approval.

There are several ways to increase the prevalence of enrollment of older people in clinical trials (20) and it is important to design clinical trials to avoid inappropriate inclusion criteria such as an arbitrary upper age limit. Previous research has shown however that the presence of criteria that may seem to be highly associated with old age, such as impaired renal or hepatic function, are not necessarily the primary reason for the exclusion of the majority of older patients from clinical trials (8). Instead, strict inclusion criteria such as requiring a specific severity of target disease, could be often the root cause of an underrepresentation of older patients in clinical trials.

It is often challenging to include older patients in clinical trials that attempt a research design to maximize patient safety whilst allowing for the potential efficacy of drugs in some therapeutic areas to be observed $(21,22)$. Even allowing for this difficulty however, in addition to being one of the most frequently used, medicines targeting the cardiovascular system are one of the most common drug classes to be inappropriately prescribed to hospitalized frail elderly patients (23). Thus, a protocol of clinical trials conducted for regulatory review of drug approval should be discussed with a regulatory agency for a better assessment of drug safety even in older population. Also, how drug safety and efficacy within more severe patients who are usually excluded from clinical trials will be assured needs to be continually discussed and evaluated as a critical issue for proper drug use in the elderly.

The high proportion of elderly-specific revisions within the therapeutic area "various" also reflects a characteristic tendency for elderly-specific revisions as most of these revisions identified potential risks that were directed toward the elderly in particular. Worryingly, the majority of these revisions were issued more than eight years after approval and were based on spontaneous reports. Especially where risks for specific geriatric syndromes are concerned, a list that includes ileus, aspiration and cognition disorder, there is a pressing need for early detection and health promotion within the elderly population through smooth implementation of postmarketing surveys and studies.

Most package insert revisions specific to the elderly were triggered by "spontaneous reports" or "regulatory action in other countries," with only a small amount of cases found to result from "others" or "more than one source." This is another issue that needs to be addressed in order to achieve better assessment of drug risk for the elderly population. In Japan, the Risk Management Plan (RMP) was introduced in April 2013 (24) and is expected to serve as a tool for healthcare professionals to gain a greater understanding of postmarketing drug safety operations, especially with regards to elderly patients. In addition, continuous benefit-risk assessment based on periodic benefit-risk evaluation reports as outlined in the ICH E2C(R2) guideline that was implemented in Japan in May 2013 (25). These will help to promote the proper use of drugs in both the elderly and non-elderly populations and to improve a quality of postmarketing safety assessment.

It should be emphasized that nearly half of the package label revisions were triggered by an increase in the known risk for elderly people. In other words, insufficient information was only available regarding the difference in the magnitude of risk between elderly and non-elderly patients at the time of drug approval, with this information only being revealed in the postmarketing phase. To identify risks in elderly patients as early as possible, pharmacometric approach which has been utilized in pediatric populations (26) could be applied. Furthermore, PMDA has initiated "MIHARI" project to implement pharmacoepidemiological approaches using electrical medical record database for safety assessment of a drug $(27,28)$. Differences in risks between elderly and non-elderly populations may be more properly characterized by the use of pharmacoepidemiological analysis. As described above, it is important that 
alternative methodologies should be more utilized in Japan for the early and better detection and evaluation of drug safety in elderly patients, especially for therapeutic area that demonstrate increased susceptibility to risk among the elderly (e.g., drug-related to the cardiovascular system).

This study had some limitations that need to be addressed. Firstly, only revisions of package inserts that were publicly available were assessed. In general, these therefore included only relatively serious concerns published by the MHLW and did not include any more minor revisions voluntarily undertaken by the MAHs. Although this was a deliberate choice in order to allow us to concentrate on the major safety issues affecting the elderly, this is something that future research will need to assess. Secondly, our categorization technique used a filter than included only revision notifications that incorporated the term "the elderly" or made specific reference to an age bracket that included those aged 65 years or over. As a result of this strict inclusion criterion, there may have been other relevant cases that were excluded from the "specific to the elderly" group. Finally, about half of the revisions explored in this study were based on spontaneous reports, whereas recognizing ADRs in the elderly is sometimes difficult (29). Considering the limited number of elderly-related revisions in this study and the fact that comorbidity and polypharmacy are common within this demographic, adverse reactions may have been underreported in some therapeutic areas. Future studies should factor such a possibility into their analysis.

\section{Conclusion}

Of all package insert revisions published in Japan, $2.1 \%$ were elderly-specific. Although there were relatively few instances of serious safety issues that arose after approval, several challenges of ensuring the safety of elderly patients in both the premarketing and postmarketing phases of release were shown in this study. It is important to more effectively utilize alternative approaches such as pharmacometrics or pharmacoeoidemiology for evaluation of drug safety in elderly patients in Japan.

This views expressed in this article are those of the authors and do not necessarily reflected the official views of the PMDA.

\section{Key points}

- Elderly-specific revisions of package inserts were frequently required for the therapeutic areas of "cardiovascular system" and "various."

- About half of all elderly-specific revisions contained an increase in the risk assessment among the elderly for safety issues that had already been identified.
- Most revisions related to safety in the elderly were triggered by spontaneous reports or regulatory action in other countries.

- It is important that alternative methodology for the early and better detection and evaluation of drug safety and efficacy should be more utilized in Japan

Acknowledgments: The authors are very grateful to Ayumi Tanaka for her editorial assistance.

Conflict of Interest: The authors declared no conflict of interest.

Ethics Statement: The authors state that no ethical approval was needed.

\section{References}

1. United Nations, Department of Economic and Social Affairs, Population Division (2011) World Population Prospects: The 2010 Revision, CD-ROM Edition. http: / / esa.un.org/wpp/Excel-Data / DB02_Stock_Indicators / WPP2010_DB2_F05_3A_OLD_AGE_DEPENDENCY_RATIO_1564.XLS. Accessed 19 August 2013.

2. Jyrkka J, Enlund H, Korhonen MJ, et al. Patterns of drug use and factors associated with polypharmacy and excessive polypharmacy in elderly persons: results of the Kuopio 75+ study: a cross-sectional analysis. Drugs \& aging. 2009; 26: 493-503. DOI: 10.2165/00002512-200926060-00006

3. Tulner LR, Kuper IM, Frankfort SV, et al. Discrepancies in reported drug use in geriatric outpatients: relevance to adverse events and drug-drug interactions. The American journal of geriatric pharmacotherapy. 2009; 7: 93-104. DOI: 10.1016/j.amjopharm.2009.04.006

4. Corsonello A, Pedone C and Incalzi RA. Age-related pharmacokinetic and pharmacodynamic changes and related risk of adverse drug reactions. Curr Med Chem. 2010; 17: 571-584. DOI: CMC - AbsEpub/ 2010 - 033 [pii]

5. Lattanzio F, Landi F, Bustacchini S, et al. Geriatric conditions and the risk of adverse drug reactions in older adults: a review. Drug safety : an international journal of medical toxicology and drug experience. 2012; 35 Suppl 1: 55-61. DOI: 10.1007/BF03319103

6. Gurwitz JH, Col NF and Avorn J. The exclusion of the elderly and women from clinical trials in acute myocardial infarction. JAMA. 1992; 268: 14171422 .

7. Zulman DM, Sussman JB, Chen X, et al. Examining the evidence: a systematic review of the inclusion and analysis of older adults in randomized controlled trials. J Gen Intern Med. 2011; 26: 783-790. DOI: 10.1007/s11606-010-1629-x

8. Asahina Y, Sugano H, Sugiyama E, et al. Representation of older patients in clinical trials for drug approval in Japan. J Nutr Health Aging. 2014; 18: 520523. DOI: $10.1007 / \mathrm{s} 12603-014-0031-5$

9. Bowie MW and Slattum PW. Pharmacodynamics in older adults: a review. Am J Geriatr Pharmacother. 2007; 5: 263-303. DOI: S1543-5946(07)00046-3 [pii] 10.1016/j.amjopharm.2007.10.001

10. Coupland C, Dhiman P, Morriss R, et al. Antidepressant use and risk of adverse outcomes in older people: population based cohort study. BMJ. 2011; 343: d4551. DOI: 10.1136/bmj.d4551 bmj.d4551 [pii]

11. Hylek EM, Evans-Molina C, Shea C, et al. Major hemorrhage and tolerability of warfarin in the first year of therapy among elderly patients with atrial fibrillation. Circulation. 2007; 115: 2689-2696. DOI: CIRCULATIONAHA.106.653048 [pii] 10.1161/ CIRCULATIONAHA.106.653048

12. ICH Harmonized Tripartite Guideline. Studies in Support of special populations: GERIATRICS E7 (1993). (Accsess Date).

13. ICH topic E7, Studies in Support of Special Populations: Geriatrics Questions and Answers (2010). (Accsess Date).

14. European Medicines Agency, Adequacy of Guidance on the Elderly Regarding Medicinal Products for Human Use (Doc. Ref. EMEA/498920/2006) (December 2006).

15. Atkin PA, Veitch PC, Veitch EM, et al. The epidemiology of serious adverse drug reactions among the elderly. Drugs \& aging. 1999; 14: 141-152.

16. Otsubo Y, Asahina Y, Noguchi A, et al. Similarities and differences between US and Japan as to pharmacogenomic biomarker information in drug labels. Drug metabolism and pharmacokinetics. 2012; 27: 142-149.

17. Uijen AA, Bakx JC, Mokkink HG, et al. Hypertension patients participating in trials differ in many aspects from patients treated in general practices. J Clin Epidemiol. 2007; 60: 330-335. DOI: S0895-4356(06)00406-9 [pii] 10.1016/j. jclinepi.2006.05.015

18. Dodd KS, Saczynski JS, Zhao Y, et al. Exclusion of older adults and women from recent trials of acute coronary syndromes. J Am Geriatr Soc. 2011; 59: 506-511. DOI: $10.1111 / j .1532-5415.2010 .03305 . x$

19. Cherubini A, Oristrell J, Pla X, et al. The persistent exclusion of older patients from ongoing clinical trials regarding heart failure. Arch Intern Med. 2011; 171: 550-556. DOI: 10.1001/archinternmed.2011.31 171/6/550 [pii] 
20. Applegate WB and Curb JD. Designing and executing randomized clinical trials involving elderly persons. Journal of the American Geriatrics Society. 1990; 38: 943-950.

21. Mody L, Miller DK, McGloin JM, et al. Recruitment and retention of older adults in aging research. Journal of the American Geriatrics Society. 2008; 56: 2340-2348. DOI: 10.1111/j.1532-5415.2008.02015.x

22. Ford JG, Howerton MW, Lai GY, et al. Barriers to recruiting underrepresented populations to cancer clinical trials: a systematic review. Cancer. 2008; 112: 228-242. DOI: 10.1002/cncr.23157

23. Hanlon JT, Artz MB, Pieper CF, et al. Inappropriate medication use among frail elderly inpatients. The Annals of pharmacotherapy. 2004; 38: 9-14. DOI: 10.1345/aph.1D313

24. Ministry of Health, Labour and Welfare (2013). Publication of Risk Management Plan. Available from: http://www.pmda.go.jp/english/ service/pdf/mhlw / PFSB-ELD-0304-1_PFSB-SD-0304-1.pdf. (Accsess Date).
25. ICH Harmonized Tripartite Guideline. PERIODIC BENEFIT-RISK EVALUATION REPORT (PBRER): E2C(R2) (2012). (Accsess Date).

26. Manolis E, Osman TE, Herold R, et al. Role of modeling and simulation in pediatric investigation plans. Paediatr Anaesth. 2011; 21: 214-221. DOI: 10.1111/j.1460-9592.2011.03523.x

27. Ikeda M., Endo A., and Matsui K. The Efforts to Utilize Electronic Medical Information for Safety Measures - PMDA's MIHARI Project - (in Japanese) Jpn J Pharmacol 2011; 16(2): DOI: http:/ / dx.doi.org/10.3820/jjpe.16.55.

28. Pharmaceutical and Medical Devices Agency. MIHARI Project -Medical Information for Risk Assessment Initiative (in Japanese); May 2014. http:/ / www.info.pmda.go.jp/kyoten_iyaku/mihari.html (accessed 1 August 2014).

29. Mannesse CK, Derkx FH, de Ridder MA, et al. Do older hospital patients recognize adverse drug reactions? Age and ageing. 2000; 29: 79-81. 